\title{
ON THE EQUIVALENCE OF LOCAL AND GLOBAL AREA-CONSTRAINT FORMULATIONS FOR LIPID BILAYER VESICLES
}

\author{
SANJAY DHARMAVARAM ${ }^{1}$ AND TIMOTHY J. HEALEY ${ }^{2}$
}

\begin{abstract}
Lipid bilayer membranes are commonly modeled as area-preserving fluid surfaces that resist bending. There appear to be two schools of thought in the literature concerning the actual area constraint. In some works the total or global area $(G A)$ of the vesicle is a prescribed constant, while in others the local area ratio is assigned to unity. In this work we demonstrate the equivalence of these ostensibly distinct approaches in the specific case when the equilibrium configuration is a smooth, closed surface of genus zero. We accomplish this in the context of the Euler-Lagrange equilibrium equations, constraint equations and the second-variation with admissibility conditions, for a broad class of models - including the phase-field type.
\end{abstract}

\section{INTRODUCTION}

Lipid membranes are commonly modeled as area-preserving fluid surfaces that resist bending. There appear to be two schools of thought in the literature concerning the actual area constraint. As in the pioneering work of Helfrich [1], the total or global area $(G A)$ of the vesicle is often a prescribed constant, e.g., cf. [2], 3], 4]. On the other hand, in analogy with the assumption of incompressibility in 3D continuum mechanics, it is natural to consider 2D-surface models characterized by local area $(L A)$ preservation, i.e., the local area ratio is assigned to unity. In this work we demonstrate the equivalence of these ostensibly distinct approaches in the specific case when the equilibrium configuration is a smooth, closed surface of genus zero. We accomplish this in the context of the Euler- Lagrange equilibrium equations, constraint equations and the second-variation with admissibility conditions, for a broad class of models - including the phase-field type.

Key words and phrases. lipid vesicles, area constraints, membrane fluidity, reparametrization invariance, conformal diffeomorphism. 
The outline of this work is as follows. In Section 2 we summarize the two formulations - LA and $G A$ - identifying their essential differences, due to distinct constraints. We note the reparametrization symmetry inherent in the $G A$ formulation in Section 3. This leads naturally to the definition of an equivalence class of solutions, each member of which has the same total surface area while satisfying the $G A$ field equations. In Section 4 we show there exists an element of the equivalence class that preserves the local area ratio, as a deformation of the unit sphere $S^{2}$. Our result here depends crucially upon the Riemann-Roch theorem, which insures that a closed, genus-zero surface in $\mathbb{R}^{3}$ is conformally equivalent to $S^{2}$, cf. [5]. With this in hand we construct an explicit change of coordinates ultimately yielding a locally area-preserving solution.

In Section 5 we consider the second-variation conditions, normally associated with the determination of local energy minima. While the expressions for the second variation agree, the area constraint equations for the two formulations again yield apparently different admissibility conditions. In particular, the pointwise condition for the $L A$ formulation involves the tangential variation, while the integral condition for the $G A$ formulation is independent of it. Employing the Hodge decomposition theorem - in this case on a genus-zero, closed surface in $\mathbb{R}^{3}$ we are able to show that the pointwise condition for the former reduces to the integral condition of the latter.

\section{Formulations}

Without loss of generality, we take the unit sphere $S^{2}$ as the reference surface of the lipid vesicle. The deformed surface of the vesicle is denoted by $\Sigma$. The deformation, $\mathbf{f}: S^{2} \rightarrow \Sigma \subset \mathbb{R}^{3}$, relates the reference and the current configurations of the membrane, i.e., $\Sigma=\mathbf{f}\left(S^{2}\right)$. In the following we employ the Einstein convention for tensors. Greek letters will be used to represent tensor indices which are assumed to lie in the set $\{1,2\}$ corresponding to surface coordinates, say, $\mathbf{x}:=\left(x^{1}, x^{2}\right)$ on $S^{2}$.

We consider a general class of phase-field models for multi-phase lipid membrane vesicles, with total internal potential energy given by

$$
\mathcal{U}(\mathbf{f}, \phi)=\int_{\Sigma} W(H, K, \phi,|\nabla \phi|) d a,
$$

where $W$ represents the energy density as a function of the mean and Gaussian curvatures fields, $H$ and $K$, respectively, on $\Sigma$, and $d a$ represents the area measure on $\Sigma$. The phase field variable $\phi$ represents the normalized difference in concentration of the lipid components. Here 
ON THE EQUIVALENCE OF LOCAL AND GLOBAL AREA-CONSTRAINT FORMULATIONS FOR LIPID BIL

$\nabla \phi$ refers to the (spatial) gradient of $\phi$ on $\Sigma$, cf. [4]. When $\phi \equiv 0$, we recover a generalized Helfrich model [1].

Since the two components do not react with each other, their total concentration is fixed on $\Sigma$. Accordingly, we impose the constraint,

$$
\int_{\Sigma}(\phi-\mu) d a=0
$$

where $\mu$ represents a fixed average concentration.

There are two common approaches to model area preservation in lipid membranes - local and global. In the former, every infinitesimal patch on the surface of the membrane preserves area under deformation [6, 7, 8]. In the other approach, the total area of the membrane is presumed fixed under deformation [9, 2, 10, 11].

2.1. Local Area (LA) Constrained Formulation. In this formulation, area preservation is modeled locally via

$$
J \equiv 1
$$

where the local area ratio $J$ is defined by $J^{2}:=\operatorname{det}\left(D \mathbf{f}^{T} D \mathbf{f}\right)$, with $D \mathbf{f}$ denoting the total surface derivative of $\mathbf{f}$.

The total potential energy can be expressed as

$\mathcal{E}_{L A}(\mathbf{u})=\int_{\Sigma} W(H, K, \phi,|\nabla \phi|) d a+\int_{S^{2}} \gamma_{L}(\mathbf{x})(J-1) d A+\lambda \int_{\Sigma}(\phi-\mu) d a-p V$

where $\gamma_{L}(\mathbf{x})$ is the Lagrange multiplier field enforcing the area constraint (2.3), $\lambda$ is the Lagrange multiplier associated with the constraint on the phase field (2.2), $p$ is the internal excess pressure and $V$ is the total volume enclosed by $\Sigma$. For notational convenience we write $\mathbf{u}:=(\mathbf{f}, \phi)$.

The Euler-Lagrange equations are obtained by taking variations of the energy (2.4) with respect to the fields $\mathbf{f}$ and $\phi$. The variation of the deformation $\mathbf{f}$ is defined by

$$
\mathbf{f}(\mathbf{x}) \mapsto \mathbf{f}(\mathbf{x})+\alpha \boldsymbol{\eta}(\mathbf{x})
$$

for sufficiently small $\alpha$.

It is standard in this field is to express the variation $\boldsymbol{\eta}$ in terms of its components normal and tangential to the surface $\Sigma$. The variation $\boldsymbol{\eta}$ when pushed forward using $\mathbf{y}=\mathbf{f}(\mathbf{x})$ takes the form

$$
\mathbf{y} \mapsto \mathbf{y}+\alpha[\mathbf{v}(\mathbf{y})+w(\mathbf{y}) \mathbf{n}(\mathbf{y})]
$$

where $\mathbf{v}$ and $w$ are the tangential and normal variations expressed in the current configuration, and $\mathbf{n}$ is the unit normal field on $\Sigma$. The 
total variation of the phase field is then of the form

$$
\phi(\mathbf{y}) \mapsto \phi(\mathbf{y})+\alpha[\nabla \phi(\mathbf{y}) \cdot \mathbf{v}(\mathbf{y})+\psi(\mathbf{y})]
$$

where $\psi$ is the spatial variation. In this way, the first variation condition can be expressed as

$$
\begin{aligned}
\delta \mathcal{E}_{L A}=\int_{\Sigma}\left\{\frac{1}{2} \Delta W_{H}+\tilde{\Delta} W_{K}+\frac{W_{\Phi}}{|\nabla \phi|} \mathrm{b}[\nabla \phi, \nabla \phi]+\right. \\
\left.\left(2 H^{2}-K\right) W_{H}+2 K H W_{K}-2 H\left[W+\gamma_{L}+\lambda(\phi-\mu)\right]-p\right\} w+ \\
\nabla \gamma_{L} \cdot \mathbf{v}+\left\{-\nabla \cdot\left(\frac{W_{\Phi}}{|\nabla \phi|} \nabla \phi\right)+W_{\phi}+\lambda\right\} \psi d a=0,
\end{aligned}
$$

for all smooth variations $\mathbf{v}, w$ and $\psi$, where $\nabla \cdot(\cdot)$ is the surface divergence and $\Delta(\cdot)$ is the Laplace-Beltrami on $\Sigma$, the various partial derivatives of $W(H, K, \phi, \Phi)$ are denoted $W_{H}=\frac{\partial W}{\partial H}$, etc., and $\tilde{\Delta}(\cdot):=\tilde{b}^{\alpha \beta} \nabla_{\alpha} \nabla_{\beta}(\cdot)$ (using the notation of [12]), $\tilde{b}^{\alpha \beta}$ being the cofactor matrix of the second fundamental form $\mathrm{b}[d \mathbf{x}, d \mathbf{x}]:=b_{\alpha \beta} d x^{\alpha} d x^{\beta}$ of $\Sigma$, i.e., $\mathrm{b}=-\nabla \mathbf{n}$.

Setting the variations $w, \mathbf{v}$ and $\psi$ pair-wise to be identically equal to zero, we obtain the following Euler-Lagrange equations,

$$
\begin{gathered}
\frac{1}{2} \Delta W_{H}+\tilde{\Delta} W_{K}+\frac{W_{\Phi}}{|\nabla \phi|} \mathrm{b}[\nabla \phi, \nabla \phi]+\left(2 H^{2}-K\right) W_{H}+2 K H W_{K} \\
-2 H\left[W+\gamma_{L}+\lambda(\phi-\mu)\right]-p=0 \\
\nabla \gamma_{L}=\mathbf{0} \\
-\nabla \cdot\left(\frac{W_{\Phi}}{|\nabla \phi|} \nabla \phi\right)+W_{\phi}+\lambda=0
\end{gathered}
$$

We recover the constraint equations by taking variations of the energy with respect to the Lagrange multipliers:

$$
\begin{gathered}
J \equiv 1, \\
\int_{\Sigma}(\phi-\mu) d a=0 .
\end{gathered}
$$

Of course (2.8b) implies that $\gamma_{L}$ is a constant over $\Sigma$.

2.2. Global Area (GA) Constrained Formulation. In this formulation, the total surface area of the membrane is assumed to be constant, viz.,

$$
\int_{\Sigma} d a=4 \pi .
$$


The total energy here is similar to that for the $L A$ formulation (discussed above), except for the term involving the area constraint, now associated with a scalar Lagrange multiplier $\gamma_{G}$ :

$$
\mathcal{E}_{G A}=\int_{\Sigma} W(H, K, \phi,|\nabla \phi|) d a+\gamma_{G} \int_{\Sigma} d a+\lambda \int_{\Sigma}(\phi-\mu) d a-p V .
$$

The first variation condition of $\mathcal{E}_{G A}$ is identical to (2.7), except the term involving the tangential variation vanishes identically. As in the $L A$ case, we then take the normal and phase-field variations and obtain the following Euler-Lagrange equations, respectively:

$$
\begin{aligned}
\frac{1}{2} \Delta W_{H}+\tilde{\Delta} W_{K}+\frac{W_{\Phi}}{|\nabla \phi|} \mathrm{b}[\nabla \phi, \nabla \phi]+\left(2 H^{2}-K\right) W_{H}+2 K H W_{K} \\
-2 H\left[W+\gamma_{G}+\lambda(\phi-\mu)\right]-p=0 \\
-\nabla \cdot\left(\frac{W_{\Phi}}{|\nabla \phi|} \nabla \phi\right)+W_{\phi}+\lambda=0 .
\end{aligned}
$$

The associated constraints are

$$
\begin{gathered}
\int_{\Sigma} d a=4 \pi \\
\int_{\Sigma}(\phi-\mu) d a=0 .
\end{gathered}
$$

Note that unlike the $L A$ formulation, the tangential equation vanishes identically in this case. We further observe that since $\gamma_{L}$ is constant on $\Sigma$ (cf. Section 22.1) the system of partial differential equations - (2.8 a , c) and $(2.11 \mathrm{a}, \mathrm{b})$ - for the two formulations are identical if we set $\gamma_{L}=\gamma_{G}=\gamma$. In the absence of the phase field $\phi$, this observation has been noted in other works [6, 13]. Although it may be tempting to deduce from this that the two formulations are equivalent, this argument alone is insufficient, due to the fact that the area constraints, (2.8d) and (2.11c), are different. Of course, any solution of the $L A$ formulation is also a solution of the $G A$ formulation.

\section{Reparametrization Symmetry}

The vanishing of the tangential equation noted above can be attributed to the reparametrization symmetry of the $G A$ formulation. This infinite-dimensional symmetry group is a manifestation of the fluidity of lipid membranes. In this section we see that as a consequence of the symmetry, solutions of the $G A$ formulation (when they exist) belong to an infinite-dimensional equivalence class. To proceed, we need to be more precise: 
By a solution of the $L A$ formulation ( $G A$ formulation), we mean there are smooth mappings $\mathbf{f}: S^{2} \rightarrow \Sigma \subset \mathbb{R}^{3}$ and $\phi: \Sigma \rightarrow \mathbb{R}$, and a smooth parametrization of $S^{2}, \mathbf{X}: \Omega \rightarrow S^{2}$, where $\Omega \subset \mathbb{R}^{2}$ is a coordinate chart, such that

$$
\mathbf{Y}\left(X^{1}, X^{2}\right):=\mathbf{f}\left(\mathbf{X}\left(X^{1}, X^{2}\right)\right)
$$

and

$$
\Psi\left(X^{1}, X^{2}\right):=\phi\left(\mathbf{Y}\left(X^{1}, X^{2}\right)\right)
$$

satisfy the system (2.8) $((2.11))$ identically. In particular, (2.8d) reads

$$
J=\sqrt{\frac{a}{A}} \equiv 1,
$$

where

$$
\begin{aligned}
& a:=\operatorname{det}\left[a_{\alpha \beta}\right], \\
& A:=\operatorname{det}\left[A_{\alpha \beta}\right],
\end{aligned}
$$

with

$$
\begin{aligned}
& a_{\alpha \beta}:=\mathbf{Y}_{, \alpha} \cdot \mathbf{Y}_{, \beta}, \\
& A_{\alpha \beta}:=\mathbf{X}_{, \alpha} \cdot \mathbf{X}_{, \beta} .
\end{aligned}
$$

being the components of the first fundamental forms for $\Sigma$ and $S^{2}$, respectively, where $\mathbf{Y}_{, \alpha}:=\frac{\partial \mathbf{Y}}{\partial X^{\alpha}}$, etc. In what follows we consistently employ the above convention, viz., the determinant of the matrix of components of the first fundamental form is denoted by the same letter employed (with indices) for the components.

Clearly, the $G A$ formulation (2.10) is independent of the coordinate parametrization of $\Sigma$, reflecting the in-plane fluidity of the model. Therefore, for any $\chi \in \operatorname{Diff}\left(S^{2}\right)$, the diffeomorphism group of $S^{2}$ into itself, we have

$$
\mathcal{E}_{G A}(\mathbf{u}(\mathbf{x}))=\mathcal{E}_{G A}(\mathbf{u} \circ \chi(\mathbf{x}))
$$

It then follows from (3.3) and the first variation condition that if $\mathbf{u}$ is a solution to the system of equations (2.11) then so is $\mathbf{u} \circ \chi$. This observation motivates the following definition.

Definition 3.1 (Equivalence class of solutions). Two solutions $\mathbf{u}$ and $\mathbf{u}^{*}$ of $G A$ formulation are said to be equivalent, if there exists a $\chi \in$ $\operatorname{Diff}\left(S^{2}\right)$ such that

$$
\mathbf{u}^{*}=\mathbf{u} \circ \chi=(\mathbf{f} \circ \chi, \phi(\mathbf{f} \circ \chi)) .
$$

We denote this equivalence class by $[\mathbf{u}]$. Clearly, this set contains infinite elements, presuming the existence of a solution. 
We remark that $\operatorname{Diff}\left(S^{2}\right)$ is not a symmetry group of the $L A$ formulation, since the local area constraint (2.8d) under reparametrizations $\chi$ transforms as

$$
J(\chi(\mathbf{x})) \operatorname{det}(D \chi(\mathbf{x}))
$$

(2.8d) is clearly not invariant unless $\operatorname{det}(D \chi(\mathbf{x})) \equiv 1$.

\section{Equivalence: Equilibria}

Let us first recall that any solution of the $L A$ formulation is automatically a solution of the $G A$ formulation and therefore, the solution set of the former is a subset of the latter. In this section, we establish a converse by showing that there is a representative in the equivalence class of the genus-zero solutions of the $G A$ formulation that satisfies the local area constraint. By explicit construction, we show that any solution $\mathbf{u}(\mathbf{x})$ of the $G A$ formulation may be mapped to a solution of the $L A$ formulation via a diffeomorphism. Since the Euler-Lagrange equations and the constraint on phase field are identical for the two formulations, it is sufficient to consider the area constraints only.

We first consider the usual parametrization of $S^{2}$ via spherical coordinates, $\left(\theta^{1}, \theta^{2}\right) \in \Omega:=(0, \pi) \times(0,2 \pi)$, viz.

$$
\mathbf{R}\left(\theta^{1}, \theta^{2}\right)=\sin \theta^{1}\left(\cos \theta^{2} \mathbf{e}_{1}+\sin \theta^{2} \mathbf{e}_{2}\right)+\cos \theta^{1} \mathbf{e}_{3},
$$

where $\left\{\mathbf{e}_{1}, \mathbf{e}_{2}, \mathbf{e}_{3}\right\}$ denotes the standard orthonormal basis for $\mathbb{R}^{3}$. The components of the first fundamental form are the given by

$$
\left[G_{\alpha \beta}\right]:=\left[\mathbf{R}_{, \alpha} \cdot \mathbf{R}_{, \beta}\right]=\left(\begin{array}{cc}
1 & 0 \\
0 & \sin ^{2}\left(\theta^{1}\right)
\end{array}\right),
$$

and thus

$$
G=\sin ^{2} \theta^{1}
$$

We assume that the current configuration, $\Sigma=\mathbf{f}\left(S^{2}\right)$, is a smooth, closed surface of genus zero and total area $4 \pi$. As in (3.1a), we consider the convected parametrization

$$
\mathbf{Y}\left(\theta^{1}, \theta^{2}\right)=\mathbf{f}\left(\mathbf{R}\left(\theta^{1}, \theta^{2}\right)\right)
$$

By virtue of the Riemann-Roch theorem [5], we know that $\Sigma$ is conformally equivalent to $S^{2}$, and without loss of generality, we assume that $\mathbf{f}(\cdot)$ is chosen in (4.3) such that

$$
g_{\alpha \beta}:=\mathbf{Y}_{, \alpha} \cdot \mathbf{Y}_{, \beta}=\lambda\left(\theta^{1}, \theta^{2}\right) G_{\alpha \beta},
$$


where $\lambda(\cdot): \Omega \rightarrow \mathbb{R}^{+}$is smooth, positive and bounded. We remark that (4.4) follows provided that $\mathbf{f}: S^{2} \rightarrow \Sigma$ is a harmonic map, cf. [5], [14]. In view of (4.2) and (4.4), we have

$$
g=\lambda^{2} G \text {. }
$$

Next we define new coordinates as follows:

$$
\Phi_{1}\left(\theta^{1}\right):=\arccos \left(1-\frac{1}{2 \pi} \int_{0}^{\theta^{1}} \int_{0}^{2 \pi} \sqrt{g}(\sigma, \tau) d \tau d \sigma\right),
$$

where, using (4.2b), (4.4) and (4.5), we have

$$
\sqrt{g}=\lambda\left(\theta^{1}, \theta^{2}\right) \sin \theta^{1} \text {. }
$$

Note that

$$
\begin{gathered}
\Phi_{1}:[0, \pi] \rightarrow[0, \pi] \text { is continuous and strictly increasing, with } \\
\qquad \Phi_{1}(0)=0 .
\end{gathered}
$$

Now from the constraint (2.11c), we have

$$
\int_{\Sigma} d a=\int_{0}^{\pi} \int_{0}^{2 \pi} \sqrt{g}(\sigma, \tau) d \tau d \sigma=4 \pi
$$

which together with (4.6) yields

$$
\Phi_{1}(\pi)=\arccos (-1)=\pi \text {. }
$$

By virtue of (4.6), we also note that

$$
\frac{d \Phi_{1}}{d \theta^{1}}=\frac{\sin \theta^{1}}{2 \pi \sin \left(\Phi_{1}\left(\theta^{1}\right)\right)} \int_{0}^{2 \pi} \lambda\left(\theta^{1}, \tau\right) d \tau>0 \text { on }(0, \pi) .
$$

Next we define

$$
\Phi_{2}\left(\theta^{1}, \theta^{2}\right):=\frac{2 \pi \int_{0}^{\theta^{2}} \lambda\left(\theta^{1}, \tau\right) d \tau}{\int_{0}^{2 \pi} \lambda\left(\theta^{1}, \tau\right) d \tau} .
$$

Clearly, for each $\theta^{1} \in[0, \pi]$ :

$\theta^{2} \mapsto \Phi_{2}\left(\theta^{1}, \theta^{2}\right)$ is continuous and strictly increasing on $[0,2 \pi]$,

$$
\begin{gathered}
\Phi_{2}\left(\theta^{1}, 0\right)=0, \text { and } \\
\Phi_{2}\left(\theta^{1}, 2 \pi\right)=2 \pi .
\end{gathered}
$$

From (4.12) we also note that

$$
\frac{\partial \Phi_{2}}{\partial \theta^{2}}=\frac{2 \pi \lambda\left(\theta^{1}, \theta^{2}\right)}{\int_{0}^{2 \pi} \lambda\left(\theta^{1}, \tau\right) d \tau}>0 \text { on }[0, \pi] \times[0,2 \pi] .
$$

Now define the coordinate change

$$
\Phi\left(\theta^{1}, \theta^{2}\right)=\left(\Phi_{1}\left(\theta^{1}\right), \Phi_{2}\left(\theta^{1}, \theta^{2}\right)\right) .
$$


ON THE EQUIVALENCE OF LOCAL AND GLOBAL AREA-CONSTRAINT FORMULATIONS FOR LIPID BIL

Since $\Phi_{1}(\cdot)$ is independent of $\theta^{2}$, we see from (4.10) and (4.13), that the Jacobian determinant of the transformation satisfies

$$
\operatorname{det}(D \Phi)=\frac{\lambda\left(\theta^{1}, \theta^{2}\right) \sin \left(\theta^{1}\right)}{\sin \left(\Phi_{1}\left(\theta^{1}\right)\right)}>0 \text { on } \Omega
$$

For our construction that follows, we need:

Lemma 4.1. $\Phi: \bar{\Omega} \rightarrow \bar{\Omega}$ is a homoeomorphism, and $\Phi: \Omega \rightarrow \Omega$ is an orientation preserving diffeomorphism.

Proof. By the construction (4.6)-(4.15), it follows that

$$
\Phi \in C\left(\bar{\Omega}, \mathbb{R}^{2}\right) \cap C^{1}\left(\Omega, \mathbb{R}^{2}\right),
$$

with positive Jacobian determinant on $\Omega$. Consider the mapping, $\tilde{\Phi}_{2}$ : $\bar{\Omega} \rightarrow \mathbb{R}$, defined by

$$
\tilde{\Phi}_{2}\left(\theta^{1}, \theta^{2}\right):=\left(1-\frac{\theta^{1}}{\pi}\right) \Phi_{2}\left(0, \theta^{2}\right)+\frac{\theta^{1}}{\pi} \Phi_{2}\left(\pi, \theta^{2}\right),
$$

and further define $\tilde{\Phi}: \bar{\Omega} \rightarrow \mathbb{R}^{2}$ via the continuous map

$$
\tilde{\Phi}\left(\theta^{1}, \theta^{2}\right):=\left(\Phi_{1}\left(\theta^{1}\right), \tilde{\Phi}_{2}\left(\theta^{1}, \theta^{2}\right)\right) .
$$

By virtue (4.8) and (4.12a,b), we note that

$$
\Phi_{1}\left(\theta^{1}\right)=0 \Longleftrightarrow \theta^{1}=0,
$$

and

$$
\tilde{\Phi}\left(0, \theta_{2}\right) \equiv \Phi_{2}\left(0, \theta_{2}\right)=0 \Longleftrightarrow \theta_{2}=0,
$$

respectively, i.e.,

$$
\tilde{\Phi}(\cdot) \text { is injective on } \bar{\Omega} \text {. }
$$

We further claim that

$$
\left.\Phi\right|_{\partial \Omega}=\left.\tilde{\Phi}\right|_{\partial \Omega} .
$$

Indeed, from (4.8), (4.9), (4.12), (4.17) and (4.18), we find:

$$
\begin{aligned}
& \tilde{\Phi}\left(0, \theta^{2}\right)=\Phi\left(0, \theta^{2}\right)=\left(0, \Phi_{2}\left(0, \theta^{2}\right)\right), \\
& \tilde{\Phi}\left(\pi, \theta^{2}\right)=\Phi\left(\pi, \theta^{2}\right)=\left(\pi, \Phi_{2}\left(\pi, \theta^{2}\right)\right), \theta^{2} \in[0,2 \pi] ; \\
& \tilde{\Phi}\left(\theta^{1}, 0\right)=\Phi\left(\theta^{1}, 0\right)=\left(\Phi_{1}\left(\theta^{1}\right), 0\right), \\
& \tilde{\Phi}\left(\theta^{1}, 2 \pi\right)=\Phi\left(\theta^{1}, 2 \pi\right)=\left(\Phi_{1}\left(\theta^{1}\right), 2 \pi\right), \theta^{1} \in[0, \pi] .
\end{aligned}
$$

With (4.15), (4.16), (4.19) and (4.20) in hand, the first assertion now follows from a well known argument based on the Brouwer degree, cf. [15. The inverse function theorem then implies that $\Phi(\cdot)$ is a local $C^{2}$-diffeomorphism, and thus it is globally so on $\Omega$. 
We now consider a new parametrization of $\Sigma$ given by

$$
\mathbf{r}\left(\phi^{1}, \phi^{2}\right):=\mathbf{Y}\left(\Phi^{-1}\left(\phi^{1}, \phi^{2}\right)\right),\left(\phi^{1}, \phi^{2}\right) \in \Omega,
$$

where $\mathbf{Y}(\cdot)$ and $\Phi(\cdot)$ are as defined in (4.3) and (4.14), respectively. Denoting the components of the first fundamental form as

$$
\left[a_{\alpha \beta}\right]:=\frac{\partial \mathbf{r}}{\partial \phi^{\alpha}} \cdot \frac{\partial \mathbf{r}}{\partial \phi^{\beta}},
$$

then direct differentiation of (4.21), using (4.4), yields

$$
\begin{gathered}
{\left[a_{\alpha \beta}\right]=D \Phi^{-T} D \mathbf{Y}^{T} D \mathbf{Y} D \Phi^{-1}} \\
=\lambda D \Phi^{-T}\left[G_{\alpha \beta}\right] D \Phi^{-1} .
\end{gathered}
$$

Taking the determinant of both sides of (4.23) leads to

$$
a=\frac{\lambda^{2} G}{(\operatorname{det} D \Phi)^{2}},
$$

and subsequent use of (4.2b) and (4.15) then gives

$$
a=\sin ^{2}\left(\phi^{1}\right) .
$$

We now state:

Theorem 4.1. Suppose that (4.3) yields a solution of the GA formulation (2.11) according to (3.1), such that the closed surface $\Sigma=\mathbf{f}\left(S^{2}\right)$ has genus zero, and thus (4.4) holds. Define the diffeomorphism $\chi$ : $S^{2} \rightarrow S^{2}$

$$
\chi:=\mathbf{R} \circ \Phi^{-1} \circ \mathbf{R}^{-1}
$$

where $\mathbf{R}(\cdot)$ and $\Phi(\cdot)$ are as defined by (4.1) and (4.14), respectively. Then

$$
\mathbf{u}^{*}:=(\mathbf{f} \circ \chi, \phi(\mathbf{f} \circ \chi))
$$

belonging to the equivalence class $[\mathbf{u}]$, cf (3.4), is a solution of the $L A$ formulation.

Proof. It is enough to show that (3.2a) is satisfied by $\mathbf{f}^{*}:=\mathbf{f} \circ \chi$. Now (4.3), (4.21) and (4.25) yield

$$
\mathbf{r}\left(\phi^{1}, \phi^{2}\right)=\mathbf{f}^{*}\left(\mathbf{R}\left(\phi^{1}, \phi^{2}\right)\right),\left(\phi^{1}, \phi^{2}\right) \in \Omega,
$$

and direct differentiation leads to

$$
\left[a_{\alpha \beta}\right]=D \mathbf{R}^{T} D \mathbf{f}^{* T} D \mathbf{f}^{*} D \mathbf{R} .
$$

Taking the determinant of both sides of (4.28) and employing (4.2b) and (4.24), we see that

$$
\operatorname{det} D \mathbf{f}^{* T} D \mathbf{f}^{*} \equiv 1
$$


ON THE EQUIVALENCE OF LOCAL AND GLOBAL AREA-CONSTRAINT FORMULATIONS FOR LIPID BIL

\section{Equivalence of Second Variation Condition}

Our next goal is to show that the second variation conditions including admissibility of variations for both formulations are equivalent. Since the problem involves constraints, specifically all smooth variations $(\mathbf{v}, w, \psi)$ (cf. Section 22.1) must satisfy the linearized constraint equations, which define admissibility. The two variants of the area constraint give us two seemingly different criteria for admissibility.

In the $L A$ formulation, the linearization of the local area constraint (2.3) is given by

$$
(\nabla \cdot \mathbf{v}-2 H w)=0 \text { on } \Sigma
$$

for all smooth variations $\mathbf{v}, w$, cf. (2.6a).

To see this, write $\mathbf{f}(\mathbf{x}) \rightarrow \mathbf{f}(\mathbf{x})+\alpha \boldsymbol{\eta}(\mathbf{x})$ for $\alpha$ sufficiently small with $\boldsymbol{\eta}(\cdot)$ smooth (as in (2.5)), and consider

$$
\operatorname{det}(D \mathbf{f}+\alpha D \boldsymbol{\eta})^{T}(D \mathbf{f}+\alpha D \boldsymbol{\eta}) \equiv 1
$$

cf. (2.3). Differentiating (5.2) with respect to $\alpha$ and then evaluation the result at $\alpha=0$ yields

$$
D \mathbf{f}^{-1} D \mathbf{f}^{-T} \cdot\left(D \mathbf{f}^{T} D \boldsymbol{\eta}+D \boldsymbol{\eta}^{T} D \mathbf{f}\right) \equiv 0 .
$$

From (2.5), (2.6a) and the chain rule we deduce

$$
D \boldsymbol{\eta}=\left(D_{\mathbf{y}} \mathbf{v}+\mathbf{n} \otimes \nabla w+w \nabla \mathbf{n}\right) D \mathbf{f},
$$

where the subscript in (5.4) is meant to emphasize the total derivative with respect to the spatial variable $\mathbf{y}$. Substituting (5.4) into (5.3) then leads to

$$
\nabla \cdot \mathbf{v}+w \nabla \cdot \mathbf{n}=0
$$

where we have used the fact that $\mathbf{n} \cdot \nabla w \equiv 0$. Finally the identity $\nabla \cdot \mathbf{n}=-2 H$ gives (5.1).

On the other hand, in the $G A$ formulation, we obtain the following linearization for the global area constraint (2.9):

$$
\int_{\Sigma}(\nabla \cdot \mathbf{v}-2 H w) d a=0
$$

From the divergence theorem, this becomes

$$
\int_{\Sigma} H w d a=0
$$

The linearization of the concentration equation (2.2) for both formulations is

$$
\int_{\Sigma}(\psi-2 H \phi w) d a=0 .
$$


Next we determine expressions for the second variation about an equilibrium configuration $\Sigma$. First consider the $L A$ formulation. We write the energy (2.4) as,

$$
\mathcal{E}_{L A}=\int_{\Sigma} \mathcal{F} d a+\int_{S^{2}} \gamma_{L}(\mathbf{x})(J-1) d A
$$

where $\mathcal{F}$ contains terms that are common to both the formulations, viz., $W, \phi, p$, etc. The second integral in the equation above accounts for the local area constraint. The first variation can be abstractly written as,

$\delta \mathcal{E}_{L A}=\int_{\Sigma} \mathcal{L}[\mathbf{v}, w, \psi] d a+\int_{\Sigma} \gamma_{L}(\nabla \cdot \mathbf{v}-2 H w) d a+\int_{S^{2}} \nu_{L}(\mathbf{x})(J-1) d A$,

where $\mathcal{L}$ is a linear operator on the variations $(\mathbf{v}, w, \psi)$ and $\nu_{L}(\mathbf{x})$ is the variation in the Lagrange multiplier field $\gamma_{L}$. The second variation then takes the form

$\delta^{2} \mathcal{E}_{L A}=\int_{\Sigma} \mathcal{B}[\mathbf{v}, w, \psi] d a+\int_{\Sigma} \gamma_{L}(\nabla \cdot \mathbf{v}-2 H w)^{2} d a+\int_{\Sigma} 2 \nu_{L}(\nabla \cdot \mathbf{v}-2 H w) d a$,

where $\mathcal{B}$ is a bilinear operator on the variations $(\mathbf{v}, w, \psi)$. By admissibility (5.1), the last integral in the previous equation vanishes. Therefore,

$$
\delta^{2} \mathcal{E}_{L A}=\int_{\Sigma} \mathcal{B}[\mathbf{v}, w, \psi]+\gamma_{L}(\nabla \cdot \mathbf{v}-2 H w)^{2} d a .
$$

In fact, the second integral in (5.12) also vanishes, but it is convenient to keep it for now.

Similarly, for the $G A$ formulation, we obtain the following expression for the second variation:

$$
\delta^{2} \mathcal{E}_{G A}=\int_{\Sigma} \mathcal{B}[\mathbf{v}, w, \psi] d a+\gamma_{G} \int_{\Sigma}(\nabla \cdot \mathbf{v}-2 H w)^{2} d a-4 \nu_{G} \int_{\Sigma} H w d a
$$

Using the admissibility condition (5.7), the last integral may be dropped and the previous equation simplifies to

$$
\delta^{2} \mathcal{E}_{G A}=\int_{\Sigma} \mathcal{B}[\mathbf{v}, w, \psi] d a+\gamma_{G} \int_{\Sigma}(\nabla \cdot \mathbf{v}-2 H w)^{2} d a .
$$

Recall (cf. Theorem 4.1) that for any equilibrium solution of the $G A$ formulation we can find an equivalent representative solution of the $L A$ formulation, and $\gamma_{L}=\gamma_{G}$ for such solutions. Accordingly we conclude that at such an equilibrium,

$$
\delta^{2} \mathcal{E}_{L A}=\delta^{2} \mathcal{E}_{G A}
$$


ON THE EQUIVALENCE OF LOCAL AND GLOBAL AREA-CONSTRAINT FORMULATIONS FOR LIPID BIL

In other words, the only difference between the second-variation conditions for the two formulations at an equilibrium is the apparently different admissibility conditions (5.1) and (5.7).

We now show that these are, in fact, the same. We first consider the 1-form, $v^{b}$ associated with $\mathbf{v}$ on $\Sigma$, defined by

$$
v^{b}:=v_{\beta} d x^{\beta},
$$

where $v_{\beta}=g_{\alpha \beta} v^{\alpha}$. Using the Hodge decomposition for the manifold $\Sigma$ [16], we write

$$
v^{b}=d \sigma+\delta \tau+\eta
$$

where $\sigma$ is a 0 -form (function), $\tau$ is a 2 -form, $\eta$ is a 1-harmonic form, $d(\cdot)$ is the exterior derivative operator and $\delta(\cdot)$ is the codifferential operator. For any vector field $\mathbf{v}$ and scalar field $\sigma$ on a 2-manifold, we note the following standard identities:

$$
\begin{gathered}
\nabla \cdot \mathbf{v}=-\delta v^{b}, \\
\Delta \sigma=-\delta d \sigma .
\end{gathered}
$$

Then using the decomposition (5.16), we write

$$
\begin{aligned}
\nabla \cdot \mathbf{v} & =-\delta(d \sigma+\delta \tau+\eta) \\
& =-\delta d \sigma=\Delta \sigma,
\end{aligned}
$$

where we have used the facts that $\delta \eta=0$ and $\delta^{2}=0$ (cf. [16]), and $(5.17 \mathrm{~b})$. By virtue of (5.18) we may rewrite the linearized local area constraint (5.1) as follows,

$$
\Delta \sigma=2 H w .
$$

From the Fredholm alternative for elliptic PDE [17], we conclude that equation (5.19) has a solution if and only if $2 \mathrm{Hw}$ is orthogonal to the null space of of the adjoint operator $\Delta$. Together with the selfadjointness of $\Delta$ and the fact that the only harmonic functions on a compact oriented Riemannian manifold are constant functions [16], we conclude that for variations $\mathbf{v}$ and $w$,

$$
\nabla \cdot \mathbf{v}=2 H w \Longleftrightarrow \int_{\Sigma} 2 H w d a=0
$$

We now conclude:

Theorem 5.1. Let the equilibrium configuration $\Sigma$ be a smooth closed surface of genus zero. Then the second variations expressions for the two formulations are the same (cf. (5.12), (5.14), (5.15)) and their admissibility conditions (5.1), (5.7) are equivalent.

An immediate consequence is: 
Corollary 5.1. With $\mathbf{u}, \mathbf{u}^{*} \in[\mathbf{u}]$ as given in Theorem 4.1, the secondvariation conditions for $\mathbf{u}$ as a solution of the $G A$ formulation are identical to those for $\mathbf{u}^{*}$ as a solution of the LA formulation.

Remark 5.1. With (5.15) and (5.20) in hand, we observe that the second term on the right sides of (5.12) and (5.14) both vanish.

\section{Concluding Remarks}

The reparametrization symmetry of the field equations for the $G A$ formulation is a reflection of the inherent in-plane fluidity of the model. This leads to a large equivalence class of equilibrium solutions. When such a solution represents a smooth, closed surface of genus zero, we demonstrate that there is a member of the equivalence class that also satisfies the field equations for the $L A$ formulation. In particular, it preserves the local area ratio as a mapping from the unit sphere to the equilibrium configuration. We then go on to show that all secondvariation conditions for these two solutions - one as solution of the $G A$ formulation and the other as a solution of the $L A$ formulation - are identical.

Questions of existence and regularity of solutions and their stability are not addressed in this work. Some progress along these lines has been made recently in [18], where a plethora of symmetry-breaking solutions for a class of phase-field models in the $G A$ formulation have been obtained. Our results here show that each solution found in [18], has a representative in the equivalence class that also satisfies the $L A$ field equations - including and especially the local area constraint.

\section{REFERENCES}

[1] Helfrich, W. 1973 Elastic properties of lipid bilayers: theory and possible experiments. Z. Naturforsch 28c, 693-703.

[2] Bonito, A., Nochetto, R. H., and Sebastian, P. M. 2010 Parametric FEM for geometric biomembranes. Journal of Computational Physics 229(9), 31713188 .

[3] Elliott, C. M. and Stinner, B. 2010 Modeling and computation of two phase geometric biomembranes using surface finite elements. Journal of Computational Physics 229(18), 6585-6612.

[4] Taniguchi, T. 1996 Shape Deformation and Phase Separation Dynamics of Two-Component Vesicles. Physical Review Letters 76(23), 4444-4447.

[5] Jost, J. 2002 Compact Riemann Surfaces. Springer-Verlag.

[6] Jenkins, J. 1973 Static equilibrium of configurations of a model red blood cell membrane. Biophysical Journal 13, 926-939.

[7] Jenkins, J. T. 1977 The equations of mechanical equilibrium of a model membrane. SIAM Journal on Applied Mathematics 32(4), 755-764. 
ON THE EQUIVALENCE OF LOCAL AND GLOBAL AREA-CONSTRAINT FORMULATIONS FOR LIPID BIL

[8] Steigmann, D. J., Baesu, E., Rudd, R., Belak, J., and McElfresh, M. 2003 On the variational theory of cell-membrane equilibria. Interfaces and Free Boundaries 5, 357-366.

[9] Elliott, C. M. and Stinner, B. 2013 Computation of Two-Phase Biomembranes with Phase Dependent Material Parameters Using Surface Finite Elements. Commun. Comput. Phys 13(2), 325-36.

[10] Feng and Klug, W. S. 2006 Finite element modeling of lipid bilayer membranes. Journal of Computational Physics 220(1), 394-408.

[11] Ma, L. and Klug, W. S. 2008 Viscous regularization and r-adaptive remeshing for finite element analysis of lipid membrane mechanics. Journal of Computational Physics 227(11), 5816-5835.

[12] Tu, Z. and Ou-Yang, Z. 2004 A geometric theory on the elasticity of biomembranes. Journal of Physics A: Mathematical and General 37(47), 11407.

[13] Steigmann, D. J. 1999 Fluid Films with Curvature Elasticity. Arch. Rational Mech. Anal. 150, 127-152.

[14] Lai, R., Wen, Z., Yin, W., Gu, X., and Lui, L. 2014 Folding-Free Global Conformal Mapping for Genus-0 Surfaces by Harmonic Energy Minimization. Journal of Scientific Computing 58(3), 705-725.

[15] Ciarlet, P. G. 1988 Mathematical Elasticity, volume 1. North-Holland.

[16] Warner, F. W. 1983 Foundations of differentiable manifolds and Lie groups. Springer-Verlag.

[17] Evans, L. C. 2010 Partial Differential Equations. Springer-Verlag.

[18] Healey, T. J. and Dharmavaram, S. 2014 Existence of Global SymmetryBreaking Solutions in an Elastic Phase-Field Model for Lipid Bilayer Vesicles. arXiv:1402.2314.

${ }^{1}$ Dept. of Mechanical and Aerospace Engineering, Cornell UniverSity, IthacA, NY., ${ }^{2}$ Dept. of Mathematics, Cornell University, IthacA, NY., 